\title{
Retrospective Review of Esophageal Carcinoma: 3-Year Experience from a Tertiary Care Teaching Institute
}

\author{
Diptajit Paul $^{1}$, Pawan Kumar ${ }^{2}$, Anil Kumar Dhull ${ }^{3}$, Rajeev Atri ${ }^{4}$, \\ Rakesh Dhankhar ${ }^{5}$, Vivek Kaushal ${ }^{6}$ \\ ${ }^{1,2}$ Junior Resident, ${ }^{3}$ Assistant Professor, ${ }^{4,5}$ Professor, ${ }^{6}$ Senior Professor and Head, \\ Department of Radiation Oncology, Pt B D Sharma PGIMS, Rohtak. \\ Corresponding Author: Diptajit Paul
}

\begin{abstract}
Introduction: Esophageal carcinoma, one of the common malignancies, generally presented in advanced stage makes these neoplasms less curable and highly lethal. Having such poor prognosis, it is significant to understand various patient and tumor facts related to treatment outcome of esophageal cancer, which varies regions wise. The present retrospective study also seeks to focus on current description of patterns and trends of tumors in esophageal cancer patients attended in a tertiary care hospital in a northern state of India and their treatment outcome.

Materials and Methods: Records of esophageal carcinoma patients over a period of 3-years were reviewed retrospectively. These records were analysed for incidence, demographic pattern, different treatment modalities and their response evaluation.

Results: A total 439-patients of esophageal carcinoma were identified. The median age at presentation was 47-years and males slightly outnumbered females. Among all the tumors, lower thoracic esophagus involvement was most predominant and most common presentation was dysphagia. Squamous cell carcinoma constituted the predominant histopathological type. Majority of patients presented in advanced stage and treated with combined modalities approach of radiation therapy, chemotherapy and surgery. Overall average survival was 13-months. Clinically, the response at last follow-up was CR in 19\%, PR and PD in 33\% each.

Conclusion: Despite its high prevalence in north India and poor survival rate, less initiative has been taken to increase awareness in preventing these cancers. Understandings of socio-demographic patterns and tumor characteristics may improve treatment outcome in these patients and improve quality of life. Further studies are needed in different regions of India, to get more treatment options which may convert the current scenario of palliative intent in to radical one in patients of esophageal carcinoma.
\end{abstract}

Keywords: Esophageal carcinoma, retrospective study, socio-demographic profile, dysphagia, squamous cell carcinoma

\section{INTRODUCTION}

Esophageal carcinoma is not among the uncommon cancer occurring in world. It has a higher incidence to mortality ratio, despite the fact that multidisciplinary approach has been taken in management of these cancers. Along with cancer management by combined modalities of surgery, radiation and chemo therapy; care of nutrition status of patient is of paramount importance to improve quality of life. To outline a good treatment strategy in patients of esophageal carcinoma, it is important to know region wise different sociodemographic profile and tumor characteristics and their impact on treatment 
Diptajit Paul et.al. Retrospective review of esophageal carcinoma: 3-year experience from a tertiary care teaching institute.

outcome. A few studies in different regions of India have already investigated these parameters in patients of esophageal cancer. The current study also focuses on patients of esophageal cancer presented in the department of radiation oncology in a tertiary care teaching hospital of Haryana to analyse their socio-demographic profile, tumor characteristics, different treatment schedules and their impact on survival rates.

\section{MATERIALS AND METHODS}

We retrospectively reviewed records of the patients with esophageal cancer who registered in Department of Radiation Oncology PGIMS Rohtak, Haryana, a tertiary care teaching hospital and regional cancer centre over a period of 3-years from January 2016 to December 2018. Records were analysed using software (MS Excel 2016) to obtain socio-demographical profile in terms of age, gender, origin, addiction status and tumor characteristics like histopathological subtypes, sites of primary lesion \& different presenting symptoms and different treatment schedules with regard to surgery, radiation therapy, chemotherapy or combined modality along with their response evaluation.

\section{RESULTS}

Total 439-patients of esophageal cancer were registered, which constituted approximately $4.2 \%$ of total cancer patients in above hospital. The age of the patients ranged from 23 to 88 years with median age at presentation being 47-years. Greater part of the cases $(53 \%)$ was seen in fifth and sixth decade of life (table 1). Overall, male $(n=300)$ predominance was seen, male to female ratio being 2.1:1. The year wise distributions among both sexes were displayed in figure 1.

\begin{tabular}{|l|l|l|l|}
\hline \multicolumn{4}{|c|}{ Table 1: Distribution of Patients according to age } \\
\hline Age groups (years) & $\mathbf{2 0 1 6}$ & $\mathbf{2 0 1 7}$ & $\mathbf{2 0 1 8}$ \\
\hline $20-29$ & 1 & 0 & 2 \\
\hline $30-29$ & 7 & 11 & 13 \\
\hline $40-49$ & 53 & 43 & 49 \\
\hline $50-59$ & 45 & 51 & 54 \\
\hline $60-69$ & 13 & 23 & 17 \\
\hline $70-79$ & 9 & 18 & 20 \\
\hline $80-89$ & 5 & 3 & 2 \\
\hline Total & 133 & 149 & 157 \\
\hline
\end{tabular}

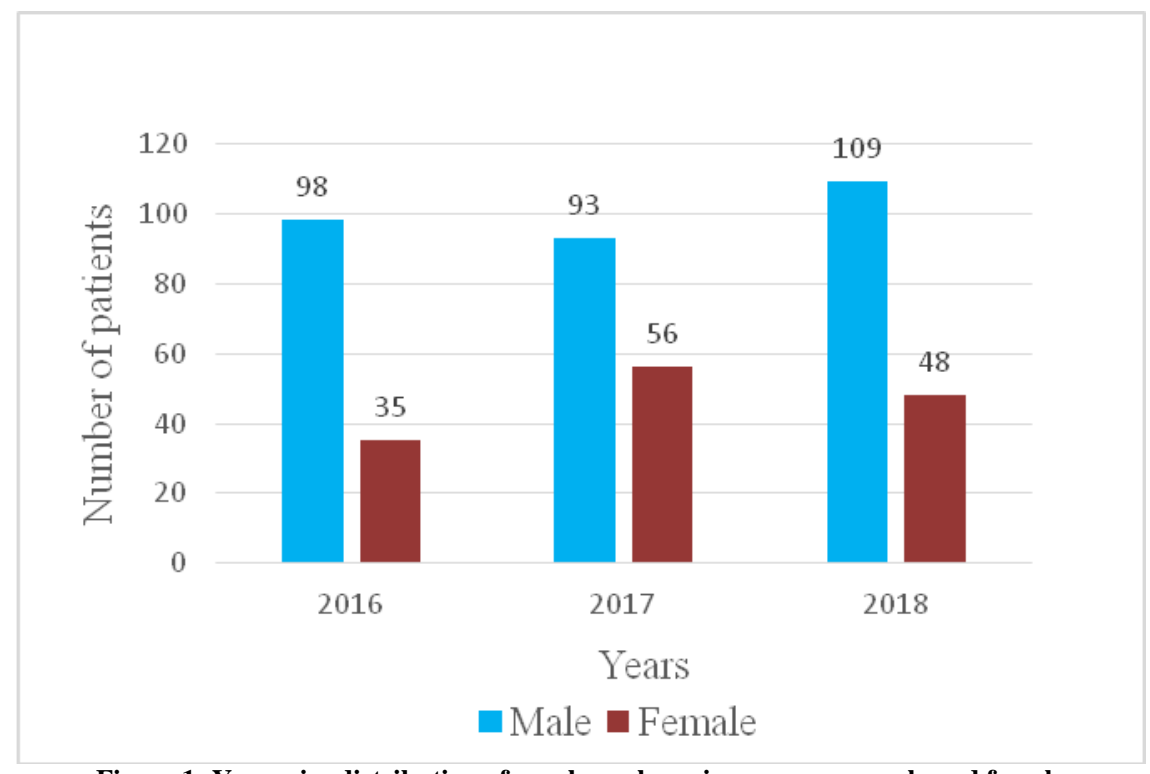

Figure 1: Year-wise distribution of esophageal carcinoma among male and female

Majority of the patients belonged to rural origin, rural to urban ratio being $4: 1$ and $70 \%(\mathrm{n}=310)$ of the total patients, irrespective of gender, were farmer. In general, $69 \%$ patients had a history of tobacco intake in the form of bidi or hookah smoking, while $47 \%$ were alcoholic; only $16 \%$ patients were recognized as having no addiction (figure 2). 
Diptajit Paul et.al. Retrospective review of esophageal carcinoma: 3-year experience from a tertiary care teaching institute.

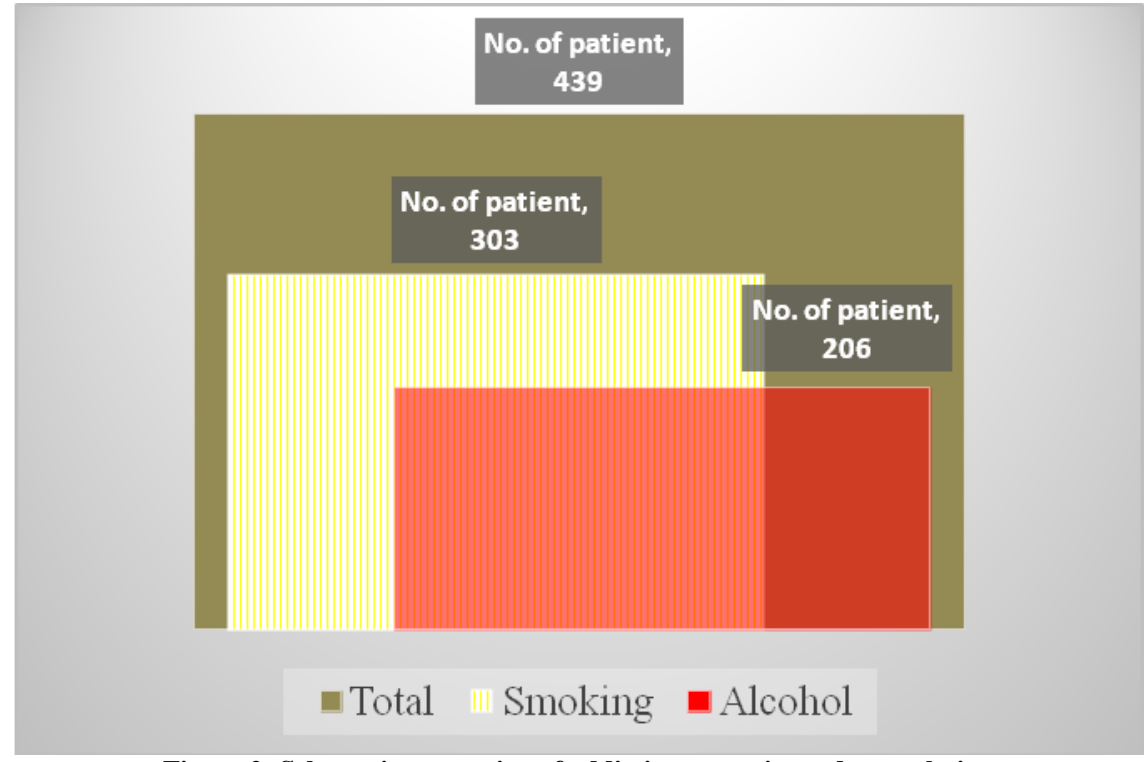

Figure 2: Schematic expression of addiction status in study population

Mean duration of symptoms was four months. Dysphagia was the most common presenting symptoms $(85 \%)$ followed by vomiting (12\%) and loss of weight $(8 \%)$. Histopathologically, squamous cell carcinoma ( $87 \%)$ constituted the largest group followed by adenocarcinoma (11\%). A few rare neoplasms $(2 \%)$ were also encountered like 4-cases of small cell neuroendocrine tumor, 3 cases of gastrointestinal stromal tumor (GIST) and 1-case each of lymphoma (esophageal mucosa-associated lymphoid tissue lymphoma) and angiosarcoma, which are treated as per rare entity clinical trial. Lower thoracic esophagus including gastroesophageal junction was the commonest site of presentation (43\%) followed by middle thoracic and cervical esophagus in $29 \%$ and $22 \%$ cases respectively (figure 3 ).

At the time of initial presentation 110-patients (25\%) were locally advanced and 97-patients (22\% of total) had metastatic disease. Most common metastatic site was liver and lungs (9\% each).

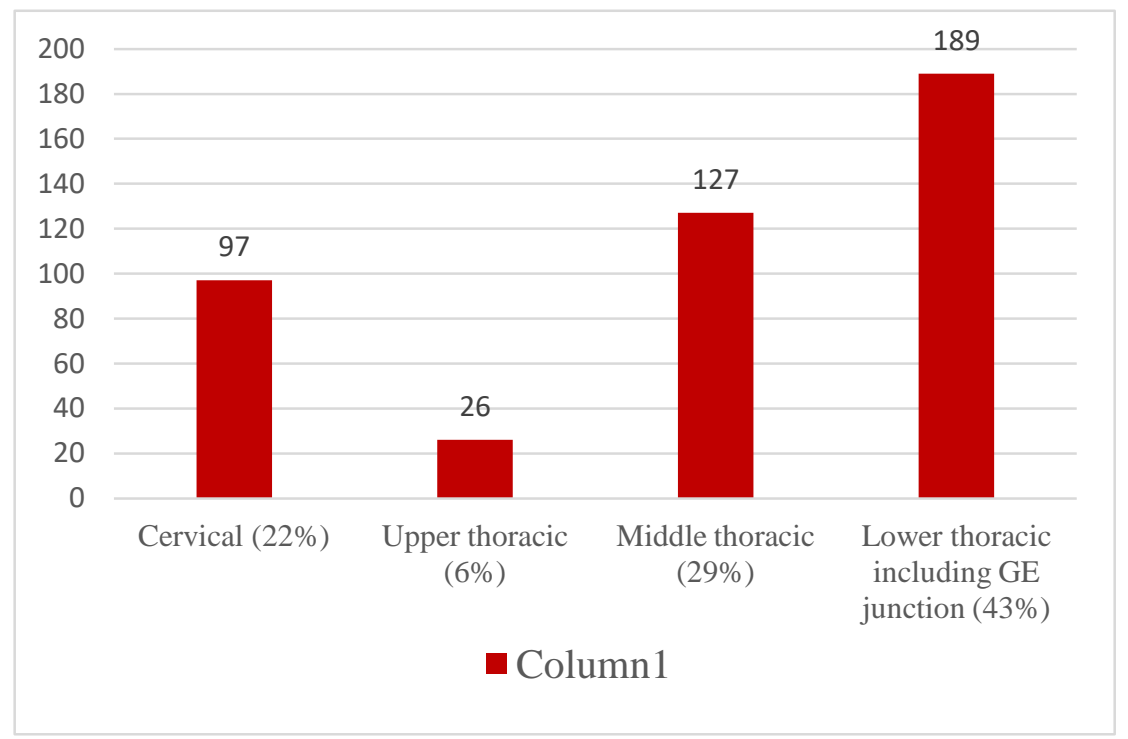

Figure 3: Site-wise distribution of esophageal cancer

The largest quantity of the patients $(n=425,97 \%)$ were pre-operative initially.
After primary evaluation, induction chemotherapy was given in $45 \%$ of the 
patients ( $\mathrm{n}=197)$ and 92-patients (21\%) received definitive concurrent chemoradiation. Among the 197-patients received induction chemotherapy, 96-patients $(22 \%$ of total patients) underwent radical surgery and 90-patients received definitive chemoradiation. 150-patients $(34 \%$ of total patients), having poor performance status, were considered for palliative therapy at initial evaluation and 124-patients among them (28\% of total patients) and 7-patients of induction chemotherapy recipient group received palliative radiotherapy. 9-patients did not report for treatment after initial evaluation and rest of the patients $(n=17$, $4 \%$ of total patients) received only palliative chemotherapy. Radical dose used predominantly was 40 gray in 20 fractions over 4-weeks. Palliative schedule was given either 20 gray in 5 fractions over 5 days, 30 gray in 10 fractions over 2-weeks or 8 gray single session mainly in very poor general condition. Most common concurrent chemotherapeutic drugs used were intravenous 5-flurouracil and platinum compound (carboplatin or cisplatin), whereas combination chemotherapy with paclitaxel plus carboplatin regimen was used in neoadjuvant and palliative only settings.

Overall average survival was 13months. Clinically the response at last follow-up was as follows: CR in 19\%, PR \& PD in 33\% each and death in 13\% patients (figure 4). In view of the progressive disease and poor general condition of the patient, $16 \%$ were given therapeutic trial with oral cyclophosphamide tablet. Among all, 74patients need feeding jejunostomy in due course of disease for survival.

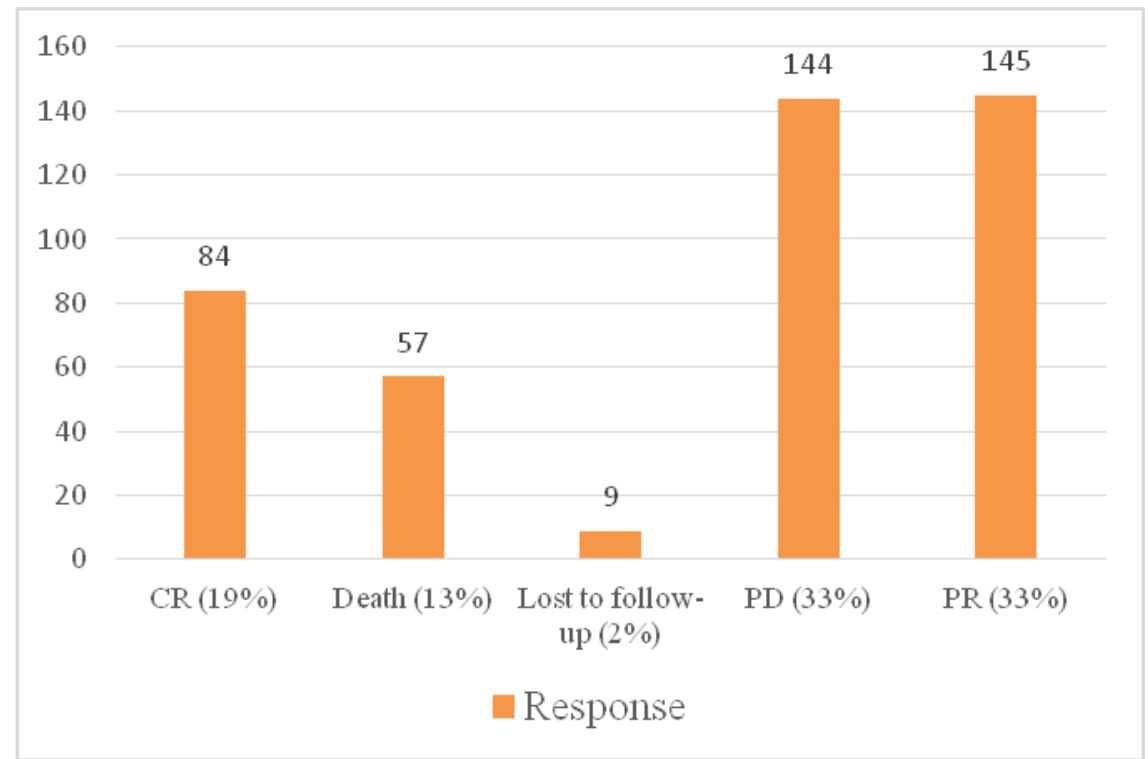

Figure 4: Response at last follow up in patients with esophageal carcinoma

\section{DISCUSSION}

Esophageal carcinoma, one of the common malignancies, creates a major concern for the oncologist due to its less curable rate. It is ninth most common cancer constituting $3.1 \%$ of all cancer cases and sixth most common cause of mortality constituting $5.5 \%$ cancer related deaths worldwide in 2020. ${ }^{[1]}$ In India esophageal cancer ranks fifth in incidence and mortality with $4.8 \%$ and $6.9 \%$ respectively in $2020 .{ }^{[2]}$ In the last few decades, there is a changing trend in the incidence and pattern of esophageal cancer. In the developed nations of western region, there is a slight decrease in total incidence of esophageal cancer and the main burden has changed from more squamous cell carcinoma to more adenocarcinoma. [3] This change in histopathological patterns attributed to certain factors, like high body mass index, obesity and increase cases of gastroesophageal reflux disease. Whereas in developing countries like India and other 
Asian countries, incidence of esophageal cancer is still in rising trends and although cases of adenocarcinoma have increased, still squamous cell carcinoma contributes the lion's share. Some rare histopathological subtypes like small cell carcinoma, sarcoma, adenoid cystic and mucoepidermoid carcinoma, melanoma and gastro-intestinal stromal tumor also occurred in esophagus with very less incidence, but clinically these are indistinguishable from typical squamous and adenocarcinoma. [4] Different risk factors, both modifiable and nonmodifiable, contribute to the development of esophageal carcinoma; most dreaded of which is smoking. Smoking is a risk factor for both squamous and adenocarcinoma, alcohol added synergistic effect to it; $90 \%$ of squamous and half of adenocarcinoma occurs due to excessive smoking. Other modifiable risk factors include chronic exposure to irritants, excessive intake of pickled vegetables, alcohol, soft drinks and spicy foods, obesity; whereas age, gender, genetical predisposition are immutable risk factors. [5] Published article on Indian population showed that socioeconomic status has a dramatic impact on esophageal cancer, poor socioeconomic status with low body mass index (BMI) contributes more to squamous cell carcinoma while rich socioeconomic status leading to high BMI and obesity causes more adenocarcinoma. [6,7] Overall, incidence of esophageal carcinoma increases with ages, people in their $6^{\text {th }}$ and $7^{\text {th }}$ decades are more affected with an appreciable male predominance and this was well proved on patients of Indian origin. $^{7}$

Progressive dysphagia is most common complaint, leading to considerable weight loss, generalized weakness. Advanced stage diseases may present with neck node involvement, hoarseness of voice, recurrent lung infection due to trachea-esophageal fistula and sometimes even with signs of distance metastasis. ${ }^{[8,4]}$ Dysphagia for long time leading to poor nutritional status is a major cause for apprehended outcome in most of these patients. At the time of initial presentation, more than half patients presented with metastatic disease, half of the rest have locally advanced disease and less than $20 \%$ presented with curable disease. ${ }^{[9]}$ Overall regional nodal involvements in esophageal cancer differ in squamous and adenocarcinoma. Cervical, thoracic and abdominal, all stations can be involved in both histology types depending upon tumor location and stage of primary lesion. [10] Common sites of distant metastasis are liver, lungs and bones; adrenal gland and brain metastasis are also seen in a few patients. ${ }^{[11]}$ Contrast enhanced computed tomography of thorax and abdomen (CECT) and endoscopic ultrasound (EUS) are the two most common diagnostic modalities having good positive predictive value in assessing accurate $\mathrm{T}$ (primary tumor) and $\mathrm{N}$ (regional node) stage as well as to guide for biopsy for tissue confirmation; whereas whole body fluorine-18-labelled fluorodeoxyglucose positron emission tomography with computed tomography (PET/CT) scan is mandatory for metastatic workup and post-chemotherapy response assessment. ${ }^{[4]}$

Management of esophageal carcinoma is a multidisciplinary approach, definitive surgery with or without chemoradiation therapy remains the curative treatment. Endoscopic resection in very early mucosa-limited lesion showed calculated 5-year survival rate of $98 \%$. $^{[12,13]}$ For resectable locally advanced lesion, esophagectomy is the mainstay of treatment, choice of transthoracic or transhiatal depends upon surgeon. [11,14] For unresectable locally advanced cases, chemoradiation is the standard of care, it may be followed by radical surgery if possible. ${ }^{[15]}$ Radiation in esophageal cancer should be given with recent more precise techniques like intensity modulated radiotherapy (IMRT), image guided radiotherapy (IGRT) and stereotactic body radiation therapy (SBRT) to spare surrounding normal tissue as much as possible. The dose ranges from 414 gray in preoperative settings to 50.4 
gray in definitive management, with 1.8 to 2 gray per fraction. [16] However, in developing countries with limited infrastructures like ours, 2-dimensional radiation by cobalt- 60 teletherapy machine of dose up to 45 gray (to limit spinal cord tolerance dose) in neoadjuvant cases is also well established. Doublet chemotherapy consists of platinum compounds (cisplatin or carboplatin) and 5-flurourcin or paclitaxel is used as concomitant chemotherapy with radical radiation. ${ }^{[17,18]}$

Chemotherapy alone is considered as secondary treatment adjunct to surgery and radiation therapy or in advanced metastatic disease or as salvage therapy in recurrent cases. For advanced and residual diseases, therapeutic trials with combination chemotherapy regimens have been tried. Different combinations of platinum compounds (cisplatin, carboplatin and oxaliplatin), taxanes (paclitaxel and docetaxel), fluoropyrimidines (fluorouracil and capecitabine) irinotecan, methotrexate, ifosfamide (with mesna coverage) and cyclophosphamide has been explored in literature with improve tumor control and increase chances of survival. ${ }^{[17,19]}$ A few targeted agents like trastuzumab in HER-2 positive adenocarcinoma, ramucirumab and bevacizumab in junctional adenocarcinoma, nivolumab \& pembrolizumab in PDL1 (programmed death-ligand 1) overexpressed tumor are well approved therapy with proved benefit. ${ }^{[20-24]}$ The role of some other targeted therapy like cetuximab, gefitinib and erlotinib, nimotozumab, panitimumab, sunitinib, entrectinib, larotrectinib in advanced, metastatic and recurrent esophageal cancer is evaluated in different studies but yet to established conclusively. [15,25-27] Patient with very advanced disease and morbid general condition sometimes only require palliative treatment.

Prognosis of esophageal carcinoma in most of the part in world is still very improbable due to advanced stage of disease at presentation and very poor tolerance to radical treatment due to poor nutrition status. Management of nutritional status is of utmost importance, in order to provide adequate anticancer therapy. Per-enteral nutrition through feeding jejunostomy is often required to provide proper nutrition.

In this retrospective analysis, male had majority of the cases and squamous cell carcinoma shared the major histological types which is well matched with similar studies done in other parts of India. ${ }^{[8,28,29]}$ Other parameters analysed in the study like background of patients (rural vs. urban), smoking habit, site of primary cancer (lower third more common) are also correlate well with previous Indian studies. ${ }^{[6-8,28,29]}$ The major limitation of this study is its retrospective nature. The follow up of these patients is continuing to get a proper idea regarding pattern of local and distal failure, which will be updated later.

\section{CONCLUSION}

In this retrospective analysis, different socio-demographic features of esophageal carcinoma patients, their tumor hallmarks, aspects of different treatment schedules had been evaluated. Overall patients were having poor prognosis and only $19 \%$ patients achieved complete response. In developing countries like India, majority of esophageal carcinoma patients presented with advanced disease and treatment outcome become disappointing. Despite radical surgery and chemo-radiation have beneficial role in complete cure of early disease; advanced stage, poor nutritional support and poor patient compliance makes the intent of treatment more palliative than radical one. In conclusion, careful monitoring of esophageal cancers in other institutions remain priorities to conclude proper nationwide demographic profile and it is recommended to establish the role of targeted therapies alone or with definitive chemo-radiation in advanced and metastatic esophageal cancer so as to prolong their survival and improve quality of life. More treatment options in future will convert the current scenario of palliative treatment to radical one in patients of esophageal cancer. 


\section{ACKNOWLEDGEMENT}

The authors would like to thank Mr. Madan Giri, record keeper of Department of Radiation Oncology, Pt B D Sharma PGIMS for his continuous efforts in keeping the records of cancer patients up-to-date and to help us providing the data for this retrospective study.

\section{Conflict of Interest: None}

\section{Source of Funding: None}

\section{REFERENCES}

1. World Health Organization International Agency for Research on Cancer (IARC). GLOBOCAN 2020: Population Fact Sheets: World, 2020 Available at https://gco.iarc.fr/today/fact-sheetspopulations Accessed 7th June, 2021

2. World Health Organization International Agency for Research on Cancer (IARC). GLOBOCAN 2020: Population Fact Sheets: India, 2020 Available at https://gco.iarc.fr/today/data/factsheets/popu lations/356-india-fact-sheets.pdf Accessed 7th June, 2021

3. Kim E, Koroukian S, Thomas Jr CR. Conditional Survival of Esophageal Cancer An Analysis from the SEER Registry (1988-2011). Journal of Thoracic Oncology. 2015 Oct 1;10(10):1490-7.

4. Chang SM, Mehta MP, Vogelbaum MA, Taylor MD, Ahluwalia MS. Neoplasms of the central nervous system. In: Devita VT Jr, Lawrence TS, Rosenberg SA, editors. Devita, Hellman and Rosenberg's cancer principles and practice of oncology. 11th ed. Philadelphia: Wolter Kluwer; 2019 p718-61

5. Kollarova $\mathrm{H}$, Machova L, Horakova D, Janoutova G, Janout V. EPIDEMIOLOGY OF ESOPHAGEAL CANCER-AN OVERVIEW ARTICLE. Biomedical Papers of the Medical Faculty of Palacky University in Olomouc. 2007 Jun 1;151(1).

6. Sehgal S, Kaul S, Gupta BB, Dhar MK. Risk factors and survival analysis of the esophageal cancer in the population of Jammu, India. Indian journal of cancer. 2012 Apr 1;49(2):245.

7. Giri PA, Singh KK, Phalke DB. Study of socio-demographic determinants of esophageal cancer at a tertiary care teaching hospital of Western Maharashtra, India. South Asian journal of cancer. 2014 Jan;3(1):54.

8. Choksi D, Kolhe KM, Ingle M, Rathi C, Khairnar H, Chauhan SG, Chaudhary V, Shukla A, Pandey V. Esophageal carcinoma: An epidemiological analysis and study of the time trends over the last 20 years from a single center in India. Journal of Family Medicine and Primary Care. 2020 Mar;9(3):1695.

9. D'Journo XB, Thomas PA. Current management of esophageal cancer. Journal of thoracic disease. 2014 May;6(Suppl 2):S253.

10. Hagens ER, van Berge Henegouwen MI, Gisbertz SS. Distribution of Lymph Node Metastases in Esophageal Carcinoma Patients Undergoing Upfront Surgery: A Systematic Review. Cancers. 2020 Jun;12(6):1592.

11. Napier KJ, Scheerer M, Misra S. Esophageal cancer: A Review of epidemiology, pathogenesis, staging workup and treatment modalities. World journal of gastrointestinal oncology. 2014 May 15;6(5):112.

12. Kato $H$, Nakajima $M$. Treatments for esophageal cancer: a review. General thoracic and cardiovascular surgery. 2013 Jun;61(6):330-5.

13. Ell C, May A, Pech O, Gossner L, Guenter E, Behrens A, Nachbar L, Huijsmans J, Vieth M, Stolte M. Curative endoscopic resection of early esophageal adenocarcinomas (Barrett's cancer). Gastrointestinal endoscopy. 2007 Jan 1;65(1):3-10.

14. Hulscher JB, van Sandick JW, de Boer AG, Wijnhoven BP, Tijssen JG, Fockens P, Stalmeier PF, ten Kate FJ, van Dekken H, Obertop H, Tilanus HW. Extended transthoracic resection compared with limited transhiatal resection for adenocarcinoma of the esophagus. New England Journal of Medicine. 2002 Nov 21;347(21):1662-9.

15. Kleinberg L, Forastiere AA. Chemoradiation in the management of esophageal cancer. Journal of Clinical Oncology. 2007 Sep 10;25(26):4110-7.

16. Gondi V, Vogelbaum MA, Grimm S, Mehta MP. Primary intracranial neoplasms. In: Halperin EC, Wazer DE, Perez CA, Brady LW, editors. Perez and Brady's principles 
and practice of radiation oncology. 7th ed. Philadelphia: Wolter Kluwer; 2019 p120643

17. Ilson DH. Esophageal cancer chemotherapy: recent advances. Gastrointestinal cancer research: GCR. 2008 Mar;2(2):85.

18. Mukherjee S, Hurt CN, Gwynne S, SebagMontefiore D, Radhakrishna G, Gollins S, Hawkins M, Grabsch HI, Jones G, Falk S, Sharma R. NEOSCOPE: A randomised phase II study of induction chemotherapy followed by oxaliplatin/capecitabine or carboplatin/paclitaxel based pre-operative chemoradiation for resectable oesophageal adenocarcinoma. European Journal of Cancer. 2017 Mar 1; 74:38-46.

19. National Comprehensive Cancer Network. Esophageal and Esophagogastric Junction Cancers (Version 2.2021) https://www.nccn.org/professionals/physicia n_gls/pdf/esophageal.pdf Accessed 7th June, 2021.

20. Davidson M, Starling N. Trastuzumab in the management of gastroesophageal cancer: patient selection and perspectives. OncoTargets and therapy. 2016;9:7235.

21. Young K, Smyth E, Chau I. Ramucirumab for advanced gastric cancer or gastrooesophageal junction adenocarcinoma. Therapeutic advances in gastroenterology. 2015 Nov;8(6):373-83.

22. Kelly RJ, Ajani JA, Kuzdzal J, Zander T, Van Cutsem E, Piessen G, Mendez G, Feliciano J, Motoyama S, Lièvre A, Uronis H. Adjuvant nivolumab in resected esophageal or gastroesophageal junction cancer. New England Journal of Medicine. 2021 Apr 1;384(13):1191-203.

23. Yamamoto S, Kato K. Pembrolizumab for the treatment of esophageal cancer. Expert Opinion on Biological Therapy. 2020 Oct 2;20(10):1143-50.

24. Ku GY, Bains MS, Do Joong Park YY, Rusch VW, Rizk NP, Yoon SS, Millang B,
Capanu M, Goodman KA, Ilson DH. Phase II study of bevacizumab and preoperative chemoradiation for esophageal adenocarcinoma. Journal of gastrointestinal oncology. 2016 Dec;7(6):828.

25. Zhang L, Ma J, Han Y, Liu J, Zhou W, Hong L, Fan D. Targeted therapy in esophageal cancer. Expert review of gastroenterology \& hepatology. 2016 May 3;10(5):595-604.

26. Liu D, Offin M, Harnicar S, Li BT, Drilon A. Entrectinib: an orally available, selective tyrosine kinase inhibitor for the treatment of NTRK, ROS1, and ALK fusion-positive solid tumors. Therapeutics and clinical risk management. 2018;14:1247.

27. Hempel D, Wieland T, Solfrank B, Grossmann V, Steinhard J, Frick A, Hempel L, Eberl T, Gaumann A. Antitumor activity of larotrectinib in esophageal carcinoma with NTRK gene amplification. The oncologist. 2020 Jun;25(6):e881.

28. Kapoor A, Kumar V, Singhal MK, Nirban RK, Beniwal SK, Kumar HS. Sociodemographic parameters of Esophageal Cancer in northwest India: A regional cancer center experience of 10 years. Indian journal of community medicine: official publication of Indian Association of Preventive \& Social Medicine. 2015 Oct;40(4):264.

29. Mustafa SA, Banday SZ, Bhat MA, Patigaroo AR, Mir AW, Bhau KS. ClinicoEpidemiological Profile of Esophageal Cancer in Kashmir. Age. 2016 Feb 1;40(7):2.

How to cite this article: Paul D, Kumar P, Dhull AK et.al. Retrospective review of esophageal carcinoma: 3-year experience from a tertiary care teaching institute. Int J Health Sci Res. 2021; 11(7): 111-118. DOI: https://doi.org/10. 52403/ijhsr.20210716 